\title{
Changes in the Mechanical Properties and Composition of Bone during Microdamage Repair
}

\author{
Gang Wang ${ }^{1,29 \uparrow}$, Xinhua Qu ${ }^{19 \uparrow}$, Zhifeng $\mathrm{Yu}^{1 *}$
}

1 Shanghai Key Laboratory of Orthopaedic Implants, Department of Orthopaedic Surgery, Shanghai Ninth People's Hospital, Shanghai Jiaotong University School of Medicine, Shanghai, China, 2 Department of Orthopedic Surgery, the Second Affiliated Hospital of Nanjing Medical University, Nanjing, China

\begin{abstract}
Under normal conditions, loading activities result in microdamage in the living skeleton, which is repaired by bone remodeling. However, microdamage accumulation can affect the mechanical properties of bone and increase the risk of fracture. This study aimed to determine the effect of microdamage on the mechanical properties and composition of bone. Fourteen male goats aged 28 months were used in the present study. Cortical bone screws were placed in the tibiae to induce microdamage around the implant. The goats were euthanized, and 3 bone segments with the screws in each goat were removed at 0 days, 21 days, 4 months, and 8 months after implantation. The bone segments were used for observing microdamage and bone remodeling, as well as nanoindentation and bone composition, separately. Two regions were measured: the first region (R1), located $1.5 \mathrm{~mm}$ from the interface between the screw hole and bone; and the second region (R2), located $>1.5 \mathrm{~mm}$ from the bone-screw interface. Both diffuse and linear microdamage decreased significantly with increasing time after surgery, with the diffuse microdamage disappearing after 8 months. Thus, screw implantation results in increased bone remodeling either in the proximal or distal cortical bone, which repairs the microdamage. Moreover, bone hardness and elastic modulus decreased with microdamage repair, especially in the proximal bone tissue. Bone composition changed greatly during the production and repair of microdamage, especially for the C (Carbon) and Ca (Calcium) in the R1 region. In conclusion, the presence of mechanical microdamage accelerates bone remodeling either in the proximal or distal cortical bone. The bone hardness and elastic modulus decreased with microdamage repair, with the micromechanical properties being restored on complete repair of the microdamage. Changes in bone composition may contribute to changes in bone mechanical properties.
\end{abstract}

Citation: Wang G, Qu X, Yu Z (2014) Changes in the Mechanical Properties and Composition of Bone during Microdamage Repair. PLoS ONE 9(10): e108324. doi:10.1371/journal.pone.0108324

Editor: Damian Christopher Genetos, University of California Davis, United States of America

Received May 26, 2014; Accepted August 20, 2014; Published October 14, 2014

Copyright: (c) 2014 Wang et al. This is an open-access article distributed under the terms of the Creative Commons Attribution License, which permits unrestricted use, distribution, and reproduction in any medium, provided the original author and source are credited.

Data Availability: The authors confirm that all data underlying the findings are fully available without restriction. All relevant data are within the paper.

Funding: This research was supported by grants from the National Natural Science Foundation for Youth (11002090), the Shanghai Natural Science Foundation (10ZR1417900), and the Program for Key Disciplines of the Shanghai Municipal Education Commission (J50206). The funders had no role in study design, data collection and analysis, decision to publish, or preparation of the manuscript.

Competing Interests: The authors have declared that no competing interests exist.

* Email: zhifengyu@gmail.com

9 These authors contributed equally to this work.

- GW and XQ are co-first authors on this work.

\section{Introduction}

The skeleton is the main weight-bearing structure in humans. Under normal conditions, loading activities result in microdamage in the living skeleton, which is repaired by bone remodeling [1]. Microdamage is primarily caused by excessive continuous fatigue loading and mechanical loading, such as that generated by implantation [2]. Compared with the microdamage caused by fatigue loading, the microdamage resulting from implant surgery has been the focus of a limited number of studies, suggesting that the implantation procedure may cause extensive microdamage to the peri-implant bone [3]. Moreover, microdamage accumulation may impair the strength of the bone-implant interface, potentially causing implant loosening; compromise the mechanical properties of bone; and increase the risk of fracture [4].

Until recently, most studies have investigated the effect of prevalent microdamage on the mechanical properties of bone. To our knowledge, there is limited data regarding the restoration of bone quality after microdamage repair. Apparently, extensive microdamage often occurs in peri-implant bone, which is affected mainly by diffuse damage. Moreover, microdamage is removed by bone remodeling and-possibly - the deposition of mineralized tissue $[5,6]$, which may change the structure and mechanical properties of local bone. The present study aimed to determine the effect of microdamage on the mechanical properties and composition of bone. Overall, we were able to confirm our hypothesis that the accumulation and repair of microdamage changes the composition of local bone, which decreases the mechanical properties of bone.

\section{Materials and Methods}

\section{Screw implantation and groups}

Fourteen skeletally mature Chinese mountain goats with a body weight ranging from $27-32 \mathrm{~kg}$ were used in the present study. The goats are all male and aged 28 months. Skeletal maturity was confirmed by radiography showing closure of the distal femoral 
and proximal tibia growth plates [7]. The goats were kept on a farm and cared for by a qualified veterinarian during the entire study. Animal Research Ethics approval was obtained from the Research Ethics Committee of the Shanghai Ninth People's Hospital. Under pentobarbital sodium (50 mg/kg, IV) anesthesia, a longitudinal incision was made along the cranial-lateral aspect of the leg. In each tibia, 5 holes were drilled into the cranial-lateral diaphysis with the centered hole located at the midline of the tibia diaphysis. The screws only passed through one side of the cortex, and the distance between neighboring screws was $20 \mathrm{~mm}$. After drilling and tapping, titanium cortical bone screws measuring $2 \mathrm{~mm}$ in diameter and $10 \mathrm{~mm}$ in length were inserted into each hole with a torque of $40 \mathrm{~N} \cdot \mathrm{cm}$. The incisions were closed in layers using 4.0 vicryl sutures. All animals were injected with tetracycline (20 mg/kg, IV), 14 days before being euthanized, for 2 consecutive days. Seven days prior to necropsy, all animals were injected with calcein $(15 \mathrm{mg} / \mathrm{kg}, \mathrm{IV})$, for 2 consecutive days. The schedules for screw implantation in each tibiae and the time of euthanasia are presented in Fig. 1. The bone segments with screws were removed and numbered according to the longitudinal direction. Segment 2 was stained with basic fuchsin, embedded in polymethyl methacrylate (PMMA) and cut cross-sectionally for the observation of microdamage and bone remodeling. Segment 4 was embedded in PMMA without basic fuchsin stain for nanoindentation testing. Segment 5 was used for bone composition testing. The samples taken at 0 days and 21 days were prepared for short-term observation, and the 4-month and 8-month samples were prepared for long-term observation. Fourteen goats were allocated to short-term $(\mathrm{n}=7)$ and long-term $(\mathrm{n}=7)$ observation separately (Fig. 1).

\section{Microdamage observation and histomorphometry measurements}

After euthanasia, the bilateral tibiae of the goats were removed, and bone segments implanted with screws were prepared for bulk staining in basic fuchsin for microdamage analysis [8]. After basic fuchsin stained, screws were removed, bone samples of $2 \mathrm{~cm}$ in length were embedded in PMMA and cut into slices with a thickness of $50 \mu \mathrm{m}$ using a hard tissue slicer (Leica SP1600, Germany). Stained sections were observed and measured under a fluorescence microscope. The Bioquant Image Analysis system (Bioquant OSTEO II VB.10.20, USA) was used to measure the porosity of the whole segments and the level of microdamage in the following 2 regions: the $\mathrm{R} 1$ region located $1.5 \mathrm{~mm}$ from the

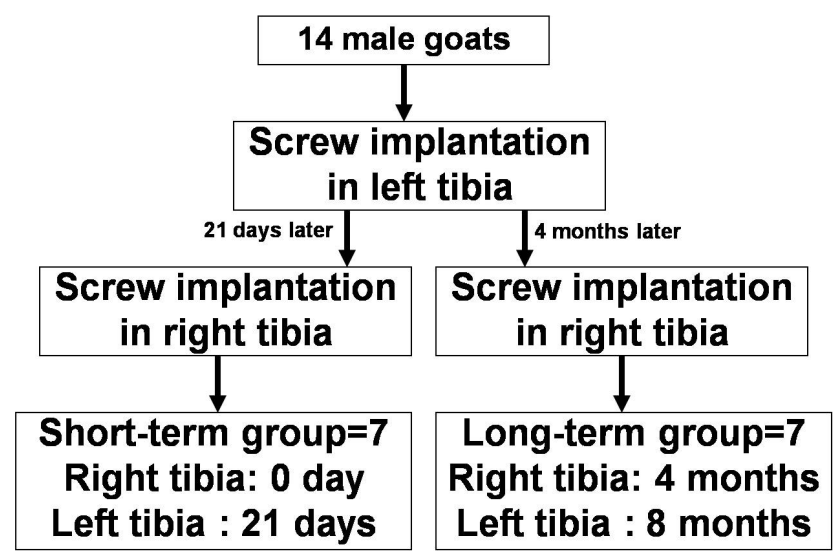

Figure 1. Schedule for screw implantation in goat tibiae in both groups.

doi:10.1371/journal.pone.0108324.g001 interface between the screw hole and the bone, and the R2 region located $>1.5 \mathrm{~mm}(1.5 \sim 3)$ from the bone-screw interface (Fig. 2) [9]. Diffuse damage often occurred in a narrow area adjacent to the bone-screw interface. In diffuse damage microcracks were too small to be discriminated by light microscopy. Therefore, pooled staining was present in bone with diffuse damage. In cross-hatched damage microcracks were vaguely distinguishable under fluorescence microscope [10]. The microdamage measurements included the damaged area in diffuse microdamage area (Dif.Dx.Ar $=$ the area of diffuse microdamage/bone area) and the crack density $(\mathrm{Cr} . \mathrm{Dn}=$ the number of cracks/bone area $)$, mean crack length (Cr.Le), and crack surface density (Cr.S.Dn = the mean crack length/bone area) in the linear cracks [11]. The variables related to bone remodeling were measured in the same region of microdamage measurement. Moreover, the densities of the cortical porosity $(\mathrm{Ct} . \mathrm{Po}=$ the number of resorption cavities/total bone area),mineralized surface (Md.S = the mineralized surface/ bone surface) and mineral apposition rate $(\mathrm{MAR}=$ interlabel distance/label interval) were calculated.

\section{Nanoindentation measurement}

The polished PMMA embedded bone blocks were examined using nanoindentation techniques as reported previously [12]. The elastic modulus $(\mathrm{E})$ and contact hardness $(\mathrm{H})$ were calculated using the Oliver-Pharr method [13], where the elastic modulus is a function of the unloading stiffness of the force-displacement curve, under the assumption that the unloading is elastic. Contact hardness is calculated as the maximum load divided by the projected area of the indenter tip at maximum load. A Nano Indenter XP system (MTS Nanoindenter XP, Oak Ridge, USA) was employed to measure the force and displacement during the indentation of the polished bone specimen. For each specimen, the sites selected for nanoindentation were consistent with the areas for microdamage measurement. Sixty-four points were selected per region. The measurement areas were determined using an optical microscope at $50 \times$ magnification. A Berkovich shape

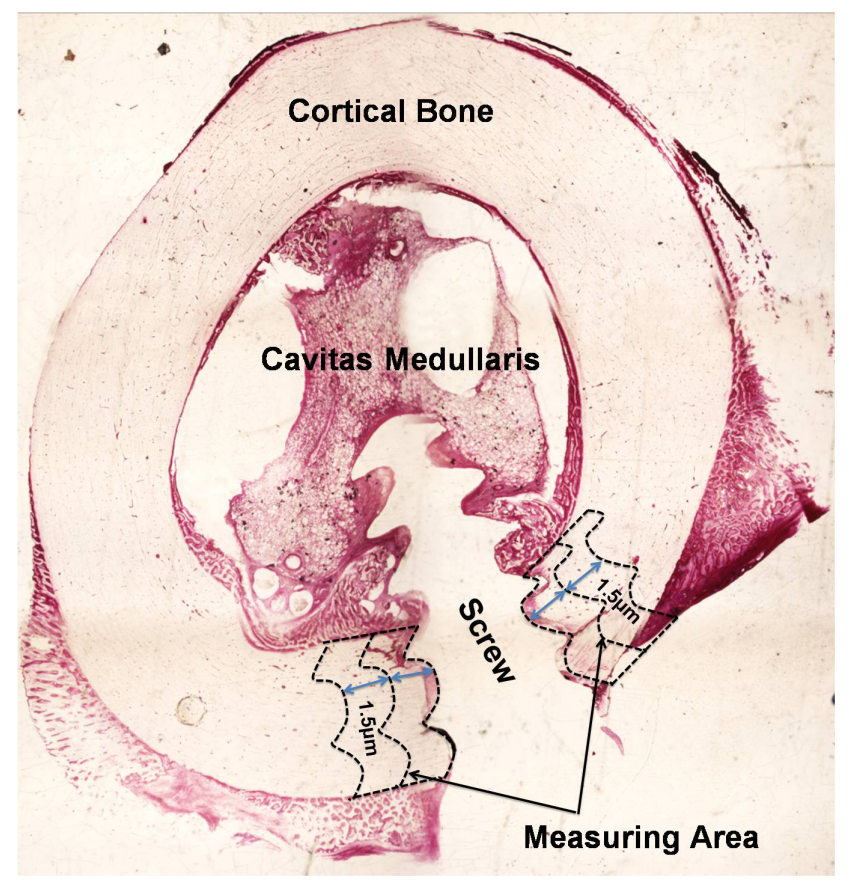

Figure 2. Schematic diagram of the measuring area. doi:10.1371/journal.pone.0108324.g002 
diamond indenter tip $(\mathrm{Ei}=1141 \mathrm{Gpa}$, vi $=0.07)$ was used to perform the nanoindentation tests at each site. The indentation procedure was under displacement control. After the surface was identified, the indenter was advanced to $500 \mathrm{~nm}$ at a speed of $10 \mathrm{~nm} / \mathrm{s}$ to avoid the effect of bone surface roughness. A typical indentation load-displacement curve includes a loading segment, a 10 -s holding period at maximum load, an unloading segment, and a 50 -s holding period for thermal drift measurement at $10 \%$ of maximum load.

\section{Bone composition measurement}

JEOL JXA-8100 electron microprobe wavelength dispersive system (WDS) was employed to detect the kinds of elements contained in the bone segment by analyzing the wavelength (or energy) of characteristic $\mathrm{X}$ ray. Backscattered electron (BSE) images were produced by Oxford Instruments INCA energy dispersive system (EDS). Then through quantitative analysis, by comparing the diffraction intensity with standard sample, quantitative result can be got from the testing sample.

\section{Statistical analyses}

All data have been tested by SPSS Statistics 18.0 (SPSS Inc., Chicago, USA) and demonstrated to have normal distributions with homogeneous variances. We used two-way analysis of variance to compare differences in microdamage, bone remodeling, nanoindentation, and bone composition between different time points in different regions. Differences between the Rl and $\mathrm{R} 2$ regions were analyzed by the paired sample $t$-test. $\mathrm{P}$ values of $<0.05$ were considered significant.

\section{Results}

\section{Microdamage morphology around the implant}

Under fluorescence microscopy, areas of microdamage dyed with basic fuchsin were observed around the implant in both regions. Diffuse microdamage and linear cracks were found in the cortical bone around the implants immediately after screw implantation ( 0 days), and diffuse microdamage was more easily observed than linear cracks in the Rl region (Fig. 3). Moreover, Fig. 3 shows that the diffuse microdamage and linear cracks were more pronounced in the $\mathrm{R} 1$ region.
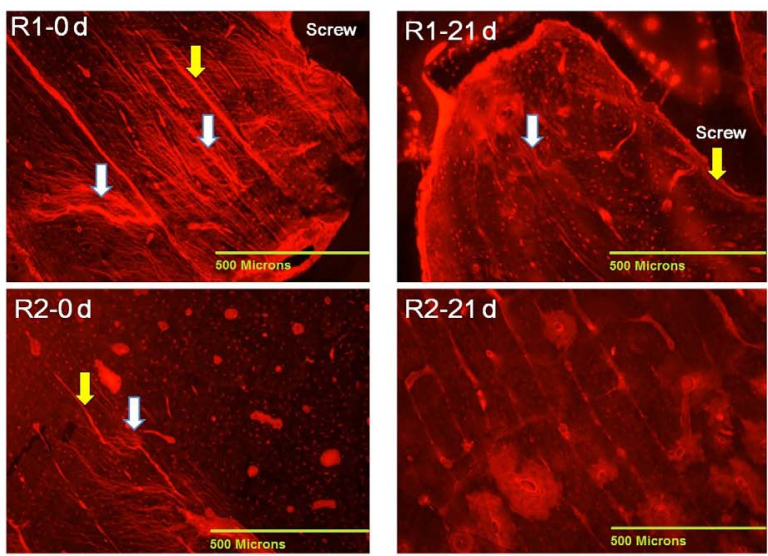

\section{Region and time differences in the microdamage} observed around the implant

We used a two-way analysis of variance to determine the region and time differences between microdamage parameters. The Dif.Dx.Ar, Cr.Dn, Cr.Le, and Cr.S.Dn in the R1 region were significantly higher than those in the $\mathrm{R} 2$ region (Fig. 4), suggesting that screw implantation can produce more diffuse microdamage and more linear cracks around the screw. Interestingly, the values of the linear cracks, especially Cr.Dn and Cr.S.Dn, are relatively higher in the $\mathrm{R} 1$ region than the $\mathrm{R} 2$ region 21 days after surgery5 -fold and 10-fold higher in the $\mathrm{R} 1$ region, respectivelyindicating that the linear cracks are more likely to be observed proximal to the screw.

\section{Repair mode for microdamage}

By comparing the results between different regions, we found no significant difference between the different regions at 0 days. At 21 days and 4 months, the labeled surface and bone porosity areas were significantly higher in the $\mathrm{R} 1$ than in the $\mathrm{R} 2$ region. However, at 8 months, although the bone porosity was higher in the $\mathrm{R} 1$ region, the labeled surface area was not different from that in the $\mathrm{R} 2$ region. Mineral apposition rate (MAR) changed greatly after surgery, especially in the $\mathrm{R} 1$ region. MAR in $\mathrm{R} 1$ region is higher than it in the $\mathrm{R} 2$ region after 21 days and 4 months of surgery. (Fig. 5).

\section{Change in micromechanical properties}

Nanoindentation testing showed that at different time points, the hardness and elastic modulus from 0 days, 21 days to 4 months showed a downward trend in both regions. Both of the hardness and elastic modulus began to increase after 4 month in R1 and R2 region. The hardness and elastic modulus of the bone tissue adjacent to the screw thread was significantly lower in the $\mathrm{R} 1$ than in the R2 region 4 months after surgery. After 8 months, the bone quality recovered slightly (Fig. 6). There was significant time difference among the 4 time points in each group $(\mathrm{P}<0.01)$.

\section{Change in bone composition}

We measured 4 elements, namely, $\mathrm{C}$ (Carbon), O (Oxygen), $\mathrm{P}$ (Phosphorus), and $\mathrm{Ca}$ (Calcium), in different regions. There was no difference between the 4 elements 0 days after surgery $(\mathrm{P}>0.05)$. At 21 days, significant differences were observed in the $\mathrm{C}, \mathrm{P}$ and $\mathrm{Ca}$ elements between the $\mathrm{R} 1$ and $\mathrm{R} 2$ regions $(\mathrm{P}<0.05)$, with $\mathrm{C}$
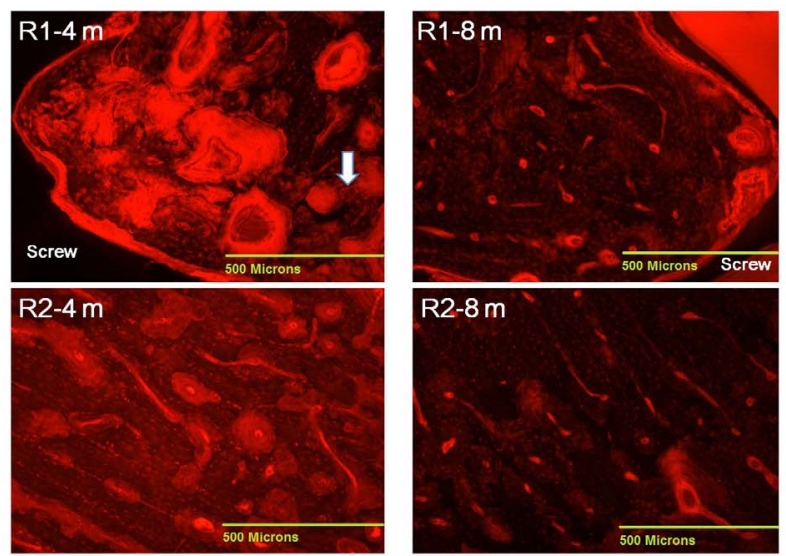

Figure 3. Microdamage accumulation and associated changes at 0 days, 21 days, 4 months, and 8 months after surgery (40x). White arrow referred to diffuse microdamage, yellow arrow referred to linear microcrack. doi:10.1371/journal.pone.0108324.g003 

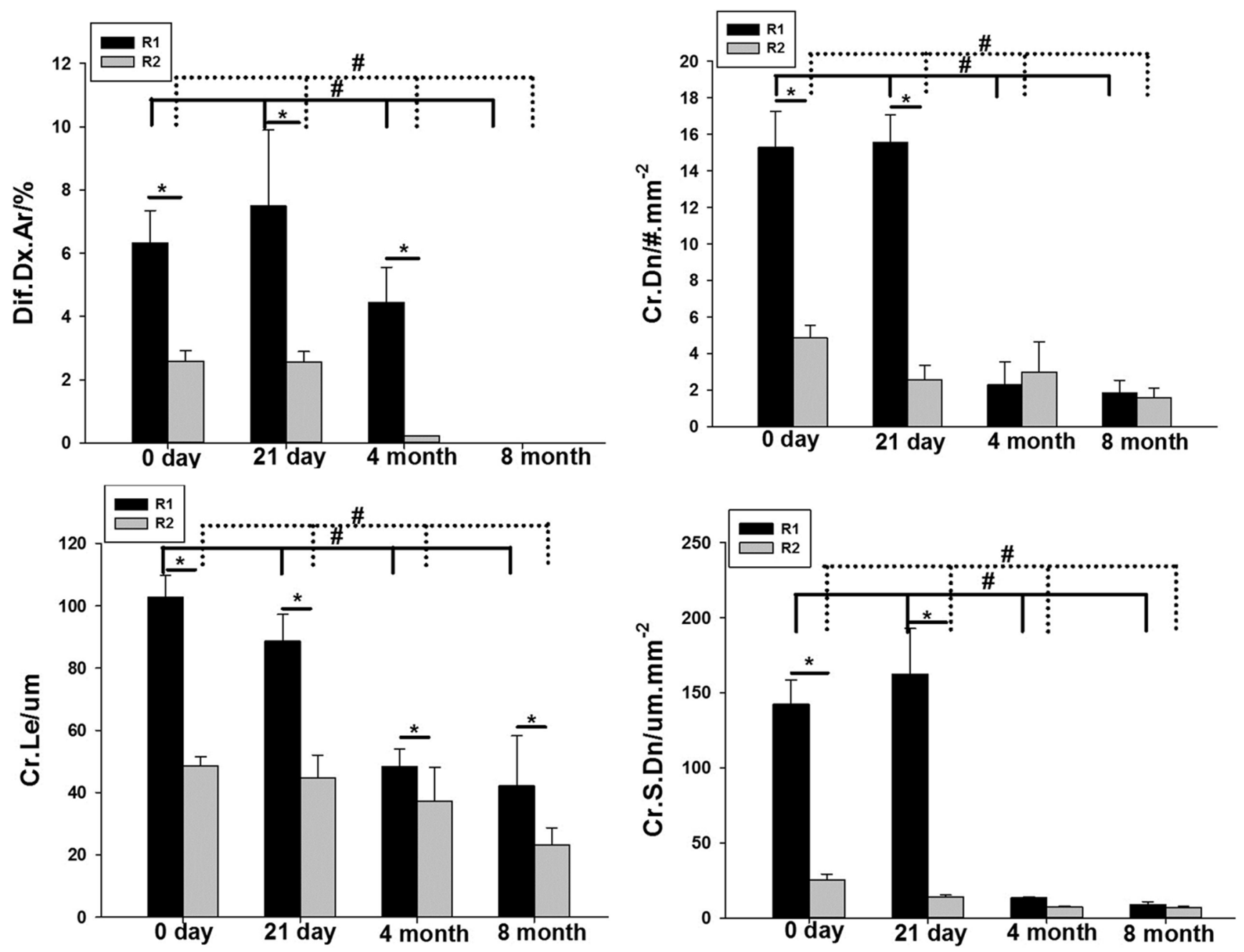

Figure 4. Production and repair of microdamage after screw implantation. ${ }^{*}$ means difference between $\mathrm{R} 1$ and $\mathrm{R} 2$ region at different time point. \# means difference between four time point in each region, solid line referred to R1 region, dotted line referred to $\mathrm{R} 2$ region. ${ }^{*}<0.0 .5$, \#< 0.05).

doi:10.1371/journal.pone.0108324.g004

being higher in the $\mathrm{R} 1$ region, and $\mathrm{P}$ and $\mathrm{Ca}$ being higher in the $\mathrm{R} 2$ region. At 4 months, $\mathrm{C}$ increased to peak levels in the $\mathrm{R} 1$ region, whereas $\mathrm{Ca}$ decreased to a minimum. At 4 months and 8 months, the $\mathrm{C}$ and $\mathrm{Ca}$ composition was significantly different between the $\mathrm{R} 1$ and $\mathrm{R} 2$ regions $(\mathrm{P}<0.01)$, whereas the $\mathrm{O}$ and $\mathrm{P}$ composition was not significantly different between groups $(\mathrm{P}<$ 0.05, Fig. 7).

\section{Discussion}

Although microdamage accumulation and increased bone remodeling are clearly evidenced in peri-screw bone $[10,14]$, the mechanisms of microdamage repair around the implant and change in the mechanical quality and composition of bone after screw insertion remain unclear. Previous studies suggest that the activation of bone resorption is considerably less effective in diffuse than in linear damage, suggesting that the response to diffuse and linear damage is different $[15,16]$. However, the large-size linear cracks are known to cause severe damage to the bone matrix, leading to osteocyte apoptosis, which initiates bone remodeling [16] and acts as the primary factor contributing to targeted bone remodeling [16]. Overall, the mechanical properties of bone and mode of microdamage repair seem to depend on the different types of microdamage.

With regard to the location of the microdamage, Wang et al. [10] reported that diffuse damage tended to appear in the area adjacent to the bone-screw interface, which seems unlikely. In the present study, we frequently observed a combination of diffuse damage and linear cracks in peri-screw bone, which was referred to as complex microdamage. However, the diffuse microdamage decreased as the distance to the implant increased, and the linear cracks were still observed. As confirmed by O'Brien et al. [17], implant surgery on the cortical bone results in increased microdamage produced near the implant, owing to the greater damage to the proximal bone. Moreover, the complex microdamage is likely to be caused by the strong impact - the drilling and cutting forces in implant surgery result in rapid damage to bone. After surgery, implant micromotion is likely to increase microdamage accumulation.

Microdamage has been postulated as a contributing factor for bone remodeling, which replaces the damaged bone with new bone. Thus, the rate of bone remodeling increased in the area with microdamage, with Wang et al. [10] which suggested that bone resorption becomes considerably active within 1 month after screw 

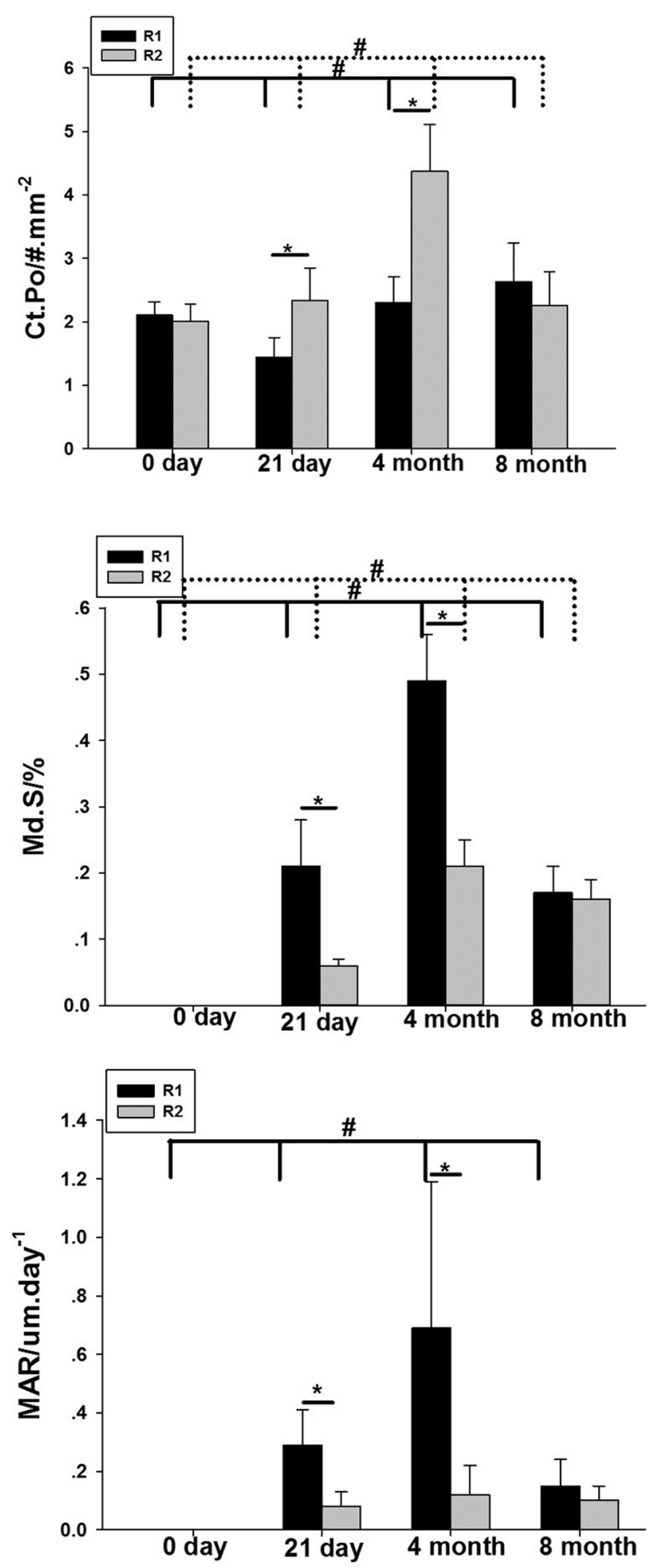

Figure 5. Comparison of bone remodeling parameters in different regions at different time points. ${ }^{*}$ means difference between R1 and R2 region at different time point. \# means difference between four time point in each region, solid line referred to $\mathrm{R} 1$ region, dotted line referred to $\mathrm{R} 2$ region. $\left.{ }^{*}<0.0 .5, \#<0.05\right)$.

doi:10.1371/journal.pone.0108324.g005 implantation. Moreover, increased bone resorption was observed which may decreased diffuse damage and linear cracks. Although screw implantation can result in different types of microdamage in cortical bone, the type of microdamage associated with activation of bone remodeling remains unclear. A previous study by Bentolila et al. [15] reported that intracortical resorption was preferentially associated with linear-type cracks, with a $40 \%$ reduction in linear microcracks occurring 10 days after fatigue loading. In contrast, diffuse microdamage did not show a significant decrease at that time. In our study, we found that the bone resorption cavity and mineralized surface increased to peak levels 4 months after surgery, while the linear crack decreased rapidly compared with that 21 days after surgery, suggesting that linear microdamage is the main contributor to activation of bone remodeling. By using bisphosphonate coating screw, Agholme et al [18] found bisphosphonate coating leads to increased bone volume around screws. This maybe caused by preventing resorption of the tissue with microdamage and provide a new way to increase implant stability. But how much bisphosphonate were released to the bone and the change of bone remodeling should be confirmed, because bisphosphonate could increases microdamage accumulation during early treatment [19].

Moreover, the accumulation of microdamage has been associated with a reduction in the elastic modulus and strength of bone tissue, and an increase in energy dissipation when the bone is loaded [1,4,17,20-22]. Although the effect of diffuse damage on bone strength remains unclear [17], the accumulation of microcracks at lower stress levels is believed increase the strength of cortical bone [23]. In fact, extensive microdamage often occurs in peri-implant bone, which is affected mainly by diffuse damage. Moreover, microdamage is removed by bone remodeling andpossibly - the deposition of mineralized tissue [5,6], which may both change the structure and mechanical properties of local bone. In the early stage of repair, multiple resorption cavities impair bone structure and act as stress concentrators, both of which reduce the mechanical properties of bone [6]. During that period, the ideal osteointegration of the implant has not been established. Our results suggest that the hardness and elastic modulus did not change significantly during the early stage. However, after 4 months, with an increase in bone remolding and decrease of microdamage, the mechanical properties of local bone began to decrease. The area located near the implant - where significant microdamage occurred - was characterized by a greater decrease in the mechanical properties of bone than the region located far away from the implant. Both of elastic modulus and hardness declined until 4 months after surgery. At the same time, bone remodeling results show that they increased significantly after surgery. The decreased bone mechanical property may be caused by newly formed bone tissue being present following resorption.

The material properties of bone are determined by the matrix composition, including minerals, collagen and water - each of which can influence the mechanical properties of bone [24]. Micropetrotic bone is characterized by the loss of osteocytes, decreased water content and high mineralization $[5,25,26]$. However, bone remodeling - although increased - is not sufficient to rapidly repair all microdamage, particularly for extensive diffuse damage. The coexistence of lower mineralized new osteonal bone and higher mineralized micropetrotic bone would further compromise the mechanical properties of bone. Accordingly, the bone quality around the endosseous implant may not return to normal immediately after microdamage repair. Moreover, increased diffuse damage may change the normal repair mode, which may compromise the restoration of bone quality after microdamage repair. By using BSE [27] and Fourier transform infrared (FTIR) 

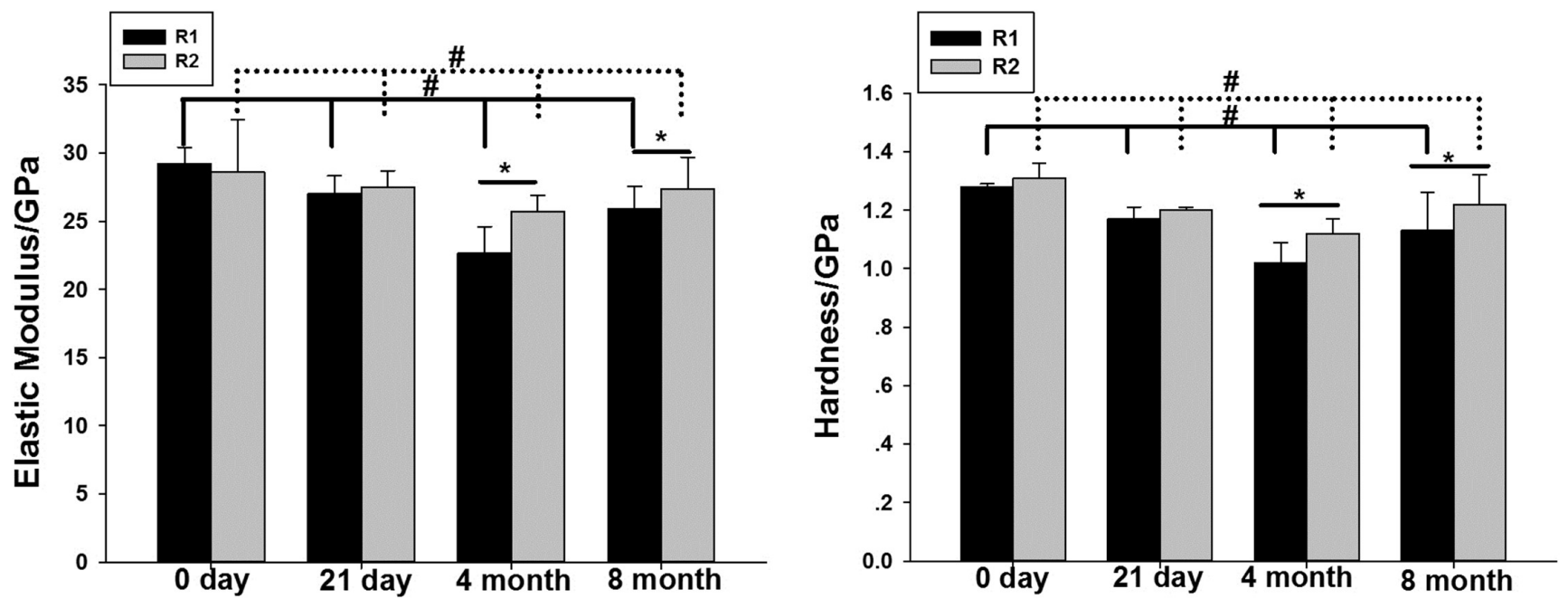

Figure 6. Comparison of nanoindentation data in different regions at different time points. * means difference between R1 and R2 region at different time point. \# means difference between four time point in each region, solid line referred to R1 region, dotted line referred to R2 region. $(*<0.0 .5, \#<0.05)$.

doi:10.1371/journal.pone.0108324.g006
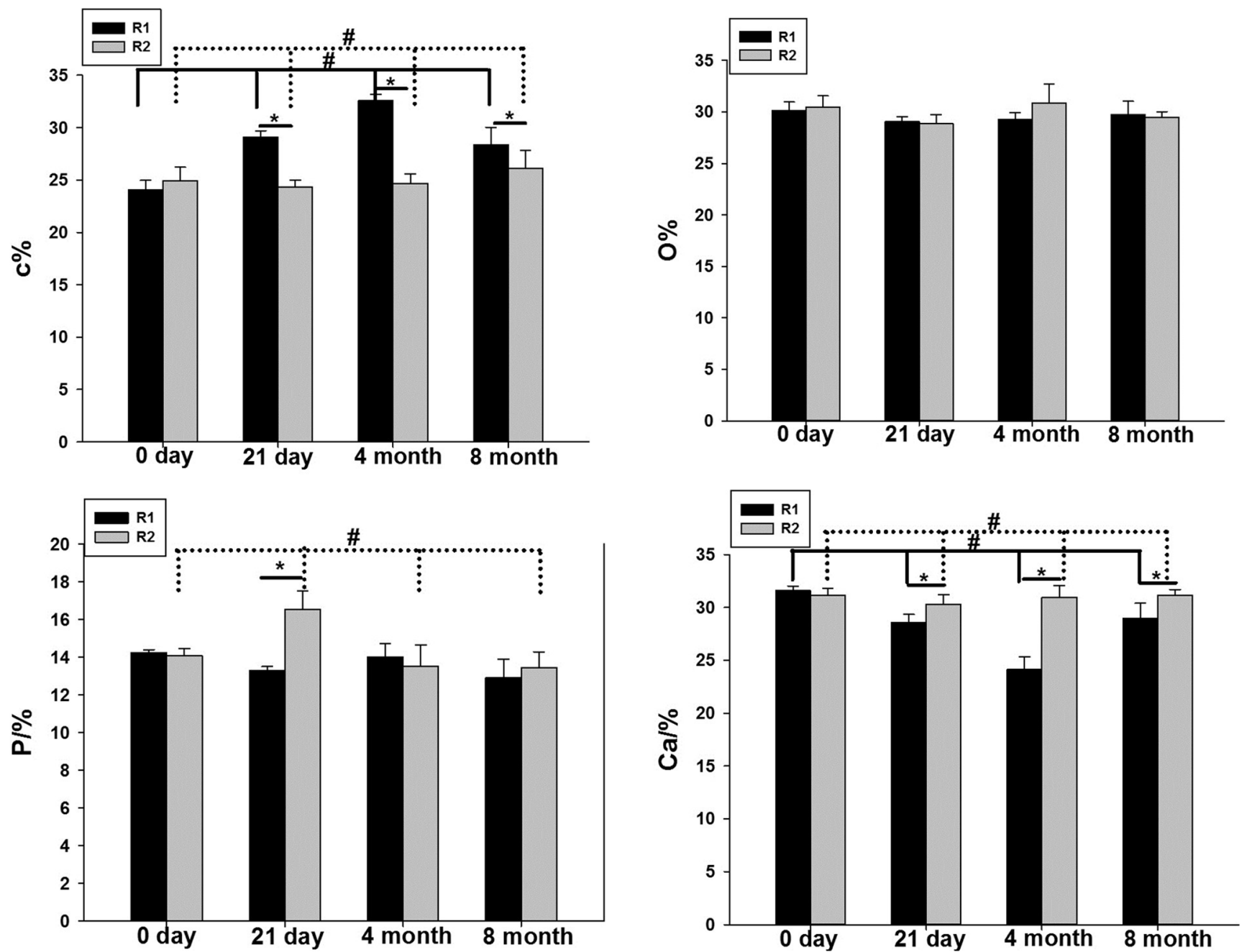

Figure 7. Comparison of bone composition $(C, O, P$, and $\mathbf{C a})$ in different regions at different time points. ${ }^{*}$ means difference between $\mathrm{R} 1$ and R2 region at different time point. \# means difference between four time point in each region, solid line referred to R1 region, dotted line referred to $\mathrm{R} 2$ region. $(*<0.0 .5, \#<0.05)$.

doi:10.1371/journal.pone.0108324.g007 
[28] imaging, previous studies have found that calcium and collagen contribute to the mechanical properties of bone. In the present study, although we did not measure changes in collagen, we did measure changes in $\mathrm{C}, \mathrm{O}, \mathrm{P}$, and $\mathrm{Ca}$. We found that 4 elements - especially $\mathrm{C}$ and $\mathrm{Ca}$ - changed greatly in the region surrounding the screw during the production and repair of microdamage. The $\mathrm{C}$ levels were highest 4 months after surgery, while the $\mathrm{Ca}$ levels were lowest at that time. Moreover, the above results suggest that bone remodeling increased to a maximum 4 months after surgery. The variation trend of bone composition may caused by bone remodeling during repair of microdamge. Change of $\mathrm{Ca}$ was the same as elastic modulus and hardness, but because the sample size is too low, we cannot find strong correlation between $\mathrm{Ca}$ and bone mechanical property.

Overall, both diffuse microdamage and linear cracks were found around the implant and accumulated during the early stage after surgery, and these types of damage may impair the initial stability of the implant. The decreased microdamage at later stages after

\section{References}

1. Martin RB (2003) Fatigue microdamage as an essential element of bone mechanics and biology. Calcif Tissue Int 73: 101-107.

2. Donahue SW, Sharkey NA, Modanlou KA, Sequeira LN, Martin RB (2000) Bone strain and microcracks at stress fracture sites in human metatarsals. Bone 27: 827-833.

3. Huja SS, Katona TR, Burr DB, Garetto LP, Roberts WE (1999) Microdamage adjacent to endosseous implants. Bone 25: 217-222.

4. Burr D (2003) Microdamage and bone strength. Osteoporos Int 14 Suppl 5: 67-72.

5. Boyde A (2003) The real response of bone to exercise. J Anat 203: 173-189.

6. Schaffler MB (2003) Role of bone turnover in microdamage. Osteoporos Int 14 Suppl 5: 73-80.

7. Leung KS, Siu WS, Cheung NM, Lui PY, Chow DH, et al. (2001) Goats as an osteopenic animal model. J Bone Miner Res 16: 2348-2355.

8. Burr DB, Hooser M (1995) Alterations to the en bloc basic fuchsin staining protocol for the demonstration of microdamage produced in vivo. Bone 17: 431433.

9. Wang L, Shao J, Ye T, Deng L, Qiu S (2012) Three-dimensional morphology of microdamage in peri-screw bone: a scanning electron microscopy of methylmethacrylate cast replica. Microsc Microanal 18: 1106-1111.

10. Wang L, Ye T, Deng L, Shao J, Qi J, et al. (2014) Repair of microdamage in osteonal cortical bone adjacent to bone screw. PLoS One 9: e89343.

11. Mashiba T, Turner CH, Hirano T, Forwood MR, Jacob DS, et al. (2001) Effects of high-dose etidronate treatment on microdamage accumulation and biomechanical properties in beagle bone before occurrence of spontaneous fractures. Bone 29: 271-278.

12. Wang X, Sudhaker Rao D, Ajdelsztajn L, Ciarelli TE, Lavernia EJ, et al. (2008) Human iliac crest cancellous bone elastic modulus and hardness differ with bone formation rate per bone surface but not by existence of prevalent vertebral fracture. J Biomed Mater Res B Appl Biomater 85: 68-77.

13. Oliver WC, Pharr GM (1992) An Improved Technique for Determining Hardness and Elastic-Modulus Using Load and Displacement Sensing Indentation Experiments. Journal of Materials Research 7: 1564-1583.

14. Yu Z, Wang G, Tang T, Fu L, Yu X, et al. (2014) Production and repair of implant-induced microdamage in the cortical bone of goats after long-term estrogen deficiency. Osteoporos Int 25: 897-903. surgery indicates that the implant-induced microdamage was repaired.The repair of microdamage by bone remodeling can change the structure and mechanical properties of local bone. Accordingly, the bone will return to normal after microdamage repair, through further remodeling activated by mechanical stimulation or other factors, which may take a relatively long time.

\section{Acknowledgments}

The present research was supported by grants from the National Natural Science Foundation for Youth (11002090), the Shanghai Natural Science Foundation (10ZR 1417900), and the Program for Key Disciplines of the Shanghai Municipal Education Commission (J50206).

\section{Author Contributions}

Conceived and designed the experiments: ZY. Performed the experiments: GW XQ ZY. Analyzed the data: XQ ZY. Contributed reagents/ materials/analysis tools: XQ ZY. Wrote the paper: ZY.

15. Bentolila V, Boyce TM, Fyhrie DP, Drumb R, Skerry TM, et al. (1998) Intracortical remodeling in adult rat long bones after fatigue loading. Bone 23: 275-281.

16. Herman BC, Cardoso L, Majeska RJ, Jepsen KJ, Schaffler MB (2010) Activation of bone remodeling after fatigue: differential response to linear microcracks and diffuse damage. Bone 47: 766-772.

17. O'Brien FJ, Brennan O, Kennedy OD, Lee TC (2005) Microcracks in cortical bone: how do they affect bone biology? Curr Osteoporos Rep 3: 39-45.

18. Agholme F, Andersson T, Tengvall P, Aspenberg P (2012) Local bisphosphonate release versus hydroxyapatite coating for stainless steel screw fixation in rat tibiae. J Mater Sci Mater Med 23: 743-752.

19. Allen MR, Burr DB (2007) Three years of alendronate treatment results in similar levels of vertebral microdamage as after one year of treatment. J Bone Miner Res 22: 1759-1765.

20. Norman TL, Yeni YN, Brown CU, Wang Z (1998) Influence of microdamage on fracture toughness of the human femur and tibia. Bone 23: 303-306.

21. Burr DB, Turner CH, Naick P, Forwood MR, Ambrosius W, et al. (1998) Does microdamage accumulation affect the mechanical properties of bone? J Biomech 31: $337-345$.

22. Schaffler MB, Radin EL, Burr DB (1989) Mechanical and morphological effects of strain rate on fatigue of compact bone. Bone 10: 207-214.

23. Sobelman OS, Gibeling JC, Stover SM, Hazelwood SJ, Yeh OC, et al, (2004) Do microcracks decrease or increase fatigue resistance in cortical bone? J Biomech 37: 1295-1303.

24. Currey JD (2002) The mechanical properties of bone. In: Currey JD, editor. Bones: Structure and mechanics. Princeton and Oxford: Princeton University Press. pp. 54-123.

25. Frost HM (1960) Micropetrosis. J Bone Joint Surg 42A: 144-150.

26. Oiu S, Sudhaker Rao D, Fyhrie DP, Palnitkar S, Parfitt AM (2005) The morphological association between microcracks and osteocyte lacunae in human cortical bone. Bone 37: 10-15.

27. Smith LJ, Schirer JP, Fazzalari NL (2010) The role of mineral content in determining the micromechanical properties of discrete trabecular bone remodeling packets. J Biomech 43: 3144-3149.

28. Brennan O, Kuliwaba JS, Lee TC, Parkinson IH, Fazzalari NL, et al. (2012) Temporal changes in bone composition, architecture, and strength following estrogen deficiency in osteoporosis. Calcif Tissue Int 91: 440-449. 